\title{
Multiple paraovarian cysts requiring emergency surgery: a rare clinical finding
}

\author{
Marie Tominaga ${ }^{1}$, Kyoko Morikawa ${ }^{1}$, Yutaro Ogawa ${ }^{1}$, Hiromi Ishiguro ${ }^{1}$, Naomi \\ Kamimura $^{1}$, Tomokazu Yokoo ${ }^{1}$, Ikunosuke Tsuneki ${ }^{1}$, Masaki Tamura ${ }^{1}$, Toru Yanase ${ }^{1}$, and \\ Takumi Kurabayashi ${ }^{1}$ \\ ${ }^{1}$ Niigata City General Hospital
}

November 14, 2021

\begin{abstract}
This report presents an unusual case of multiple paraovarian cysts that required emergency surgery due to a paraovarian cyst being entrapped by another paraovarian cyst. Laparoscopic surgery is considered useful for diagnostic and therapeutic purposes and is therefore recommended owing to difficulty in differentiating paraovarian cysts from ovarian cysts.
\end{abstract}

Manuscript prepared for submission to Clinical Case Reports

Multiple paraovarian cysts requiring emergency surgery: a rare clinical finding

Marie Tominaga $^{1 *}$, Kyoko Morikawa $^{1}$, Yutaro Ogawa ${ }^{1}$, Hiromi Ishiguro ${ }^{1}$, Naomi Kamimura ${ }^{1}$, Tomokazu Yokoo $^{1}$, Ikunosuke Tsuneki ${ }^{1}$, Masaki Tamura ${ }^{1}$, Toru Yanase ${ }^{1}$, Takumi Kurabayashi ${ }^{1}$

${ }^{1}$ Department of Obstetrics \& Gynecology, Niigata City General Hospital, Niigata, Japan.

* Correspondence should be addressed to: Marie Tominaga,

Department of Obstetrics \& Gynecology

Niigata City General Hospital

Niigata, Japan.

Tel: $+81(25)$ 281-5151

E-mail:marie.tominaga@gmail.com

Data availability statement: Not applicable

Funding statement: Not applicable

Conflict of interest disclosure: The authors declare that they have no current financial arrangement or affiliation with any organization that may have a direct influence on their work.

Patient consent statement: Written informed consent was obtained from the patients for the publication of their information and imaging.

Keywords: paraovarian cyst, ovarian cyst, mature cystic teratoma, torsion, laparoscopy

Key clinical message: This report presents an unusual case of multiple paraovarian cysts that required emergency surgery due to a paraovarian cyst being entrapped by another paraovarian cyst. Laparoscopic 
surgery is considered useful for diagnostic and therapeutic purposes and is therefore recommended owing to difficulty in differentiating paraovarian cysts from ovarian cysts.

A 38-year-old woman was brought to our hospital with left lower abdominal pain. She had been diagnosed with a left ovarian cyst approximately 10 years ago, and had undergone observation at another hospital.

An emergency computed tomography (CT) scan revealed a $55 \mathrm{~mm}$ ovarian cyst with suspected torsion of the left ovary tube (Fig. 1). Laparoscopic examination revealed enlargement of the left ovary with no evidence of torsion. There were four paraovarian cysts near the left fallopian tube with a cyst entrapped by another that appeared necrotic.

The ovarian and paraovarian cysts were removed (Fig. 2a-d). The pathological findings revealed the ovarian cysts to be benign mature cystic teratomas, and the paraneoplastic ovarian cysts to be benign serous cysts (Fig. 3). The postoperative course was uneventful, with no recurrence.

Paraovarian/paratubal cysts constitute about $10 \%$ of adnexal masses that are usually asymptomatic and rarely cause torsion. ${ }^{1}$ These cysts have no pedicle, and when they cause torsion, the ovary, fallopian tube, and infundibulopelvic ligament are often involved. ${ }^{2}$

This is a first report of entrapment of a paraovarian cyst by another. Preoperative diagnosis was challenging; however, laparoscopy was useful for diagnostic and therapeutic purposes.

Authorship: All the authors made substantial contribution to the preparation of this manuscript and approved the final version for submission.

MT, KM, YO: drafted the manuscript;

MT: corresponding author;

HI, NK, TY, IT, MT and TY: clinical support;

TK: careful review of the manuscript.

\section{References}

Kiseli M, Caglar G, Cengiz S, Karadag D, Yılmaz M. Clinical diagnosis and complications of paratubal cysts: review of the literature and report of uncommon presentations. Arch. Gynecol. Obstet. 2012; 285(6): 1563-1569.

Durairaj A, Gandhiraman K. Complications and management of paraovarian cyst: a retrospective analysis. J. Obst. Gynecol. India. 2018;69(2):180-184.

\section{Figure legends}

Figure 1. Computed tomography imaging findings

(a) Transverse computed tomography (CT) and (b) Coronal CT images demonstrate 55-mm cystic lesions with calcification and fluid formation in the left ovary (red arrowheads). The presence of torsion is unknown.

Figure 2. Intraoperative photographs

1. Image of an ovarian cyst being enucleated. The left ovary is enlarged to 5-6 $\mathrm{cm}$ (white arrow) with no evidence of torsion.

2. (Far view image) There are four paraovarian cysts near the left fallopian tube, one of which is entrapped by another (white arrow).

3. (Near view image) The entrapped paraovarian cyst has a purple discoloration and is suspected to be necrotic (white arrow). The remaining paraneoplastic ovarian cysts and ovarian cysts do not show torsion.

4. The ovarian and paraovarian cysts are removed. 
Figure 3. Macroscopic and pathological findings

(a) Macroscopic findings of ovarian cysts. (b-d) Pathological examination shows mature cystic teratoma (Skin appendages, adipose tissue, airway epithelium, and bone tissue are present). (c) Macroscopic findings of paraovarian cysts. (d) Pathological examination shows cysts lined by serous epithelium. No malignant characteristics were found.

\section{Figure 1}
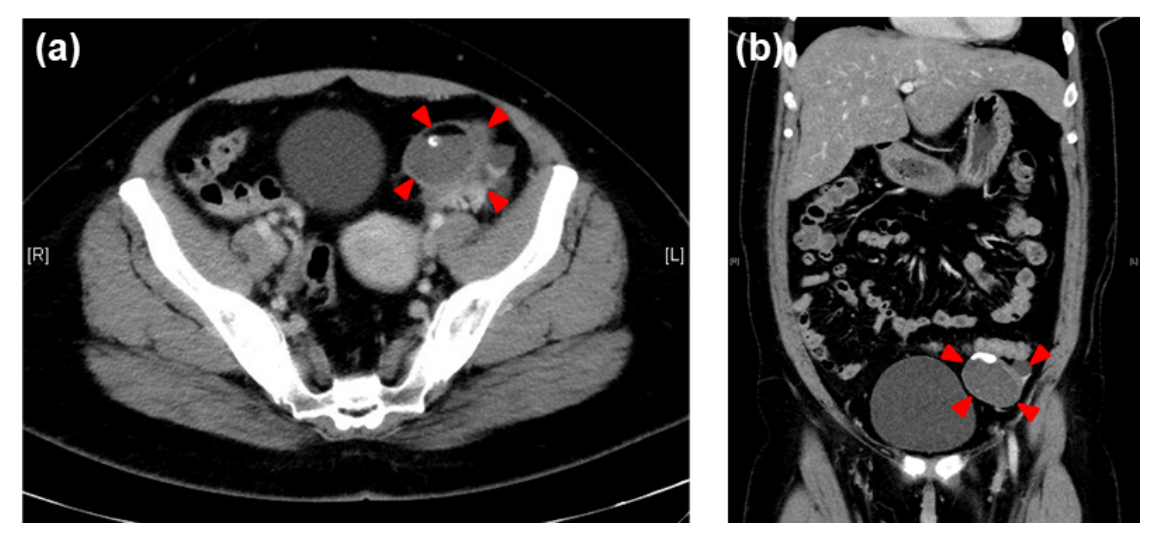

Figure 2
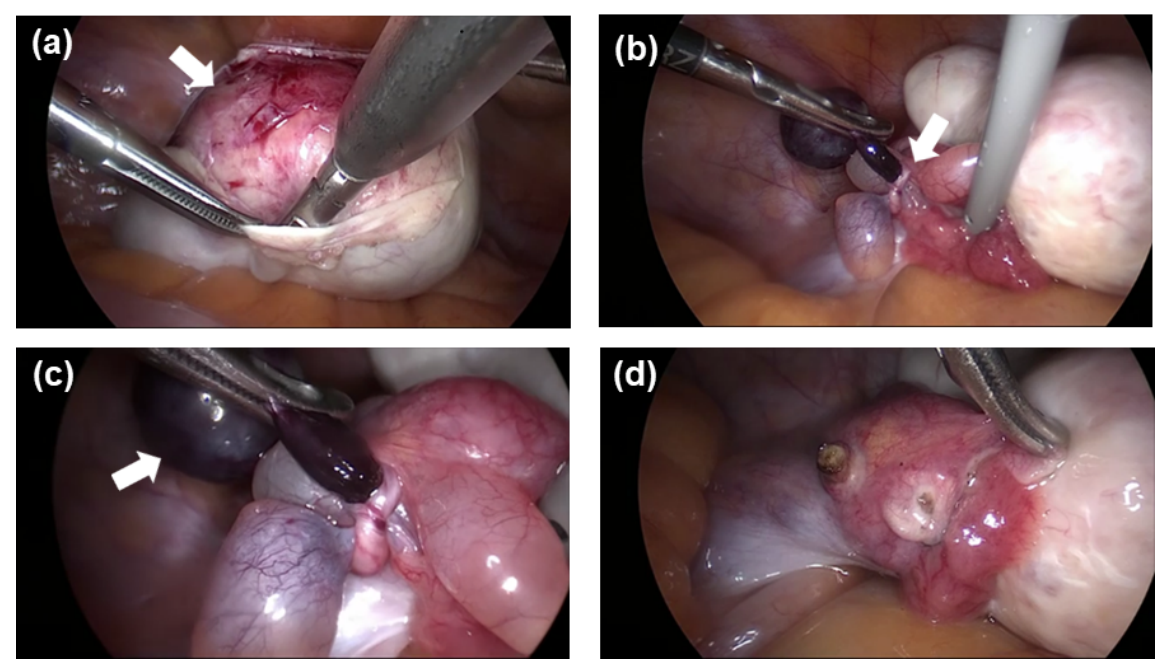
Figure 3
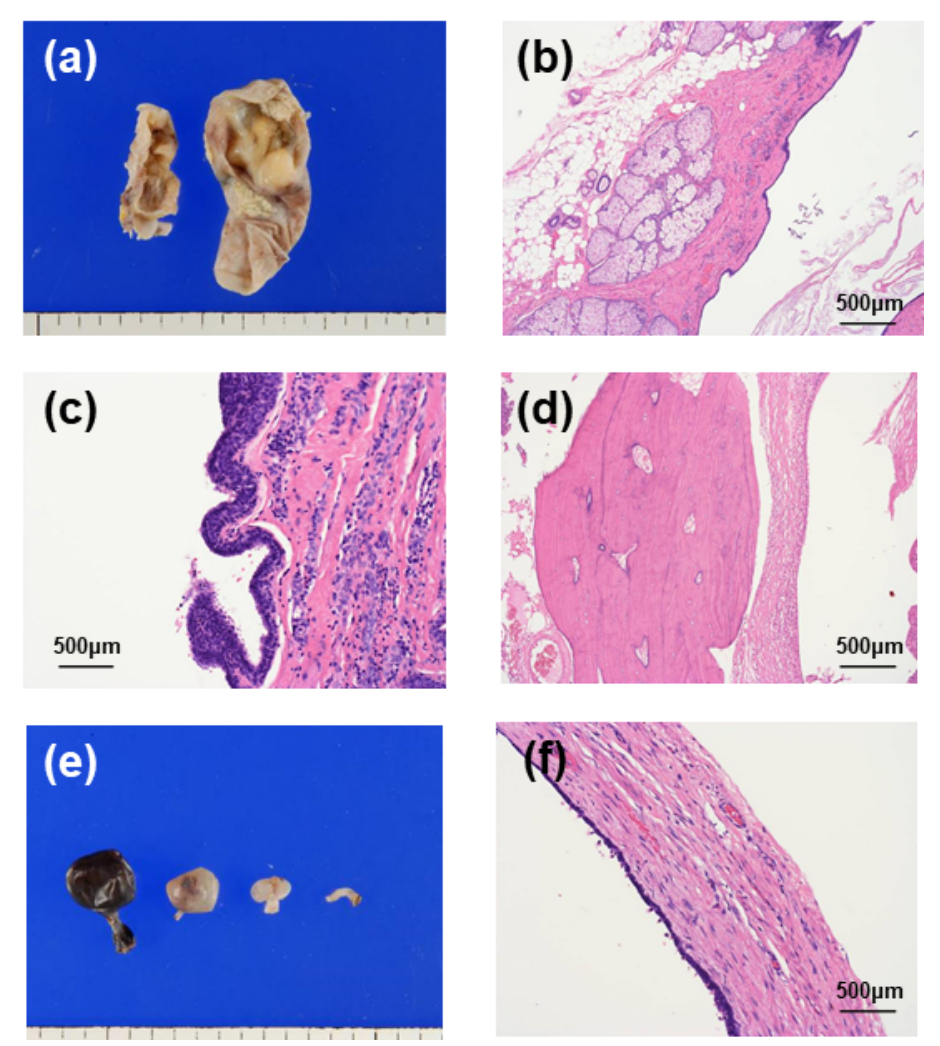